\title{
Dynamic changes in cold hardiness, high-temperature tolerance and trehalose content in the onion maggot, Delia antiqua (Diptera: Anthomyiidae), associated with the summer and winter diapause
}

\author{
Michikazu Nomura and Yukio Ishikawa \\ Laboratory of Applied Entomology, Graduate School of Agricultural and Life Sciences, The University of Tokyo, Tokyo 113- \\ 8657, Japan
}

(Received 24 July 2000; Accepted 18 June 2001)

\begin{abstract}
Changes in cold hardiness, high-temperature tolerance and trehalose content in the onion maggot, Delia antiqua (Diptera: Anthomyiidae), associated with the summer and winter diapause were investigated. Survival of summer- and winter-diapausing pupae after a 15 day exposure to $-15^{\circ} \mathrm{C}$ was greater than $80 \%$, while less than $5 \%$ of non-diapausing pupae survived the same treatment. Greater than $80 \%$ of winter-diapausing pupae survived $-23^{\circ} \mathrm{C}$ for 15 days, but survival of summer-diapausing pupae at the same procedure was less than $30 \%$. More than $75 \%$ of summer- and winter-diapausing pupae, but not non-diapausing pupae, tolerated $35^{\circ} \mathrm{C}$ for 15 days. Trehalose concentrations in summer- and winter-diapausing pupae were not high at the beginning of diapause $(\approx 5 \mu \mathrm{g} / \mathrm{mg})$, but increased gradually and reached $10 \mu \mathrm{g} / \mathrm{mg}$ in 40 days, although they were kept at relatively high temperatures of $25^{\circ} \mathrm{C}$ (summer diapause) or $15^{\circ} \mathrm{C}$ (winter diapause). Cold hardiness of pupae, however, was not proportional to their trehalose content; although trehalose concentrations in winter- and summer-diapausing pupae at day 40 (WD40 and SD40) were similar $(\approx 10 \mu \mathrm{g} / \mathrm{mg})$, cold hardiness of these pupae differed largely (WD40>SD40).
\end{abstract}

Key words: Delia antiqua, cold hardiness, high-temperature tolerance, trehalose, diapause

\section{INTRODUCTION}

The onion maggot, Delia antiqua (Diptera: Anthomyiidae), has 3-4 generations a year in Japan, and is known to overwinter as diapausing pupae (Tomioka, 1977). Furthermore, the summer population of this species is suggested to pass the hottest season as diapausing pupae (Maki et al., 1959; Park et al., 1990). In the laboratory, winter diapause in $D$. antiqua is induced by low temperatures and short day-lengths $\left(\leq 15^{\circ} \mathrm{C}\right.$ under $12 \mathrm{~L}-$ 12D, Ishikawa et al., 1987), and summer diapause is induced by high temperatures and long daylengths $\left(\geq 23^{\circ} \mathrm{C}\right.$ under $16 \mathrm{~L}-8 \mathrm{D}$, Ishikawa et al., 2000). Many insect species are known to enter both summer and winter diapause, however, a majority of these species enter summer and winter diapause at different stages (Masaki, 1980). Therefore, although a comparison of summer and winter diapause is useful to probe the nature of diapause, relevant comparisons are possible in a limited number of species that enter the two types of diapause at the same stage, e.g., D. antiqua and the cabbage armyworm, Mamestra brassicae (Kimura and
Masaki, 1992; Goto and Hukushima, 1995).

One of the features of diapause is the tolerance for extreme environmental conditions (Tauber et al., 1986). Winter-diapausing insects generally show strong cold hardiness. The increase of cold hardiness in some insects is tightly linked to diapause, whereas in others it occurs coincidentally but independently from diapause (Denlinger, 1991). Some insects in summer diapause show enhanced tolerance for high-temperature and desiccation, although experimental analyses of the association between diapause and tolerance are scanty (Tauber et al., 1986). In D. antiqua, the changes of cold hardiness and high-temperature tolerance associated with winter and summer diapause remained to be studied, although the dynamics of these processes have been investigated in the cabbage maggot, $D$. radicum, a congener of $D$. antiqua (Koštál and Šimek, 1995).

Many insects accumulate sugars and/or polyols, e.g., trehalose, sorbitol and glycerol, as cryoprotectants during overwintering. Accumulation of these compounds is triggered by low temperature and/or by entering winter diapause itself (Storey and 
Storey, 1983; Han and Bauce, 1995). In many instances, accumulation of cryoprotectants is associated with, and plays an important role in the enhancement of cold hardiness in winter-diapausing insects. In contrast, accumulation of sugars and polyols in summer-diapausing insects is not well documented. In D. radicum, trehalose was shown to be the major cryoprotectant (Koštál and Šimek, 1995). In our preliminary experiments with $D$. antiqua, a significant increase of trehalose, but not glycerol, was observed in winter-diapausing pupae (Yamashita, unpublished data).

In yeast, trehalose is indicated to be involved in tolerance for multiple stresses, e.g., freezing, heat, salt and desiccation (D'amore et al., 1991; Eleutherio et al., 1993; Soto et al., 1999). Recently, expression of foreign trehalose-6-phosphate synthase gene was shown to confer desiccation tolerance on human cells and tobacco plants (Romero et al., 1997; Guo et al., 2000). It is therefore possible that trehalose is involved in high-temperature and desiccation tolerance, as well as cold hardiness, in insects. If this is the case, summer- and winter-diapausing pupae are expected to show cross-tolerance to cold and high temperature, respectively.

In the present study, survival of $D$. antiqua pupae in summer diapause, those in winter diapause and those not in diapause was examined after exposure to subzero temperatures $(-15$ or $\left.-23^{\circ} \mathrm{C}\right)$ or to high temperature $\left(35^{\circ} \mathrm{C}\right)$ for different periods. We also investigated whether the accumulation of trehalose is associated with the summer and winter diapause in D. antiqua.

\section{MATERIALS AND METHODS}

Insects. Onion maggots were originally collected at Sapporo, Japan, and their offspring have been reared on an artificial diet (Ishikawa et al., 1983) for about 30 generations in the laboratory. Adults were reared in a cage $(30 \times 30 \times 30 \mathrm{~cm})$ with a supply of water, sugar and yeast extract (Dried Yeast Extract D-3, Wako). The cages were kept in an environmental room controlled at $23 \pm 1^{\circ} \mathrm{C}$ and under a photocycle of $16 \mathrm{~h}$ light- $8 \mathrm{~h}$ dark $(16 \mathrm{~L}-8 \mathrm{D})$ and $50-70 \%$ relative humidity. A plastic dish $(8 \mathrm{~cm}$ diam. $\times 4 \mathrm{~cm}$ height) containing damp glass beads and several pieces of onion was placed in the cage as the oviposition substrate. Insects were reared under the following conditions to induce summer or winter diapause (Ishikawa et al., 1987, 2000). In the present study, the age of pupae was expressed by the days after pupariation, with the day of pupariation being day 0 .

1) Winter diapause (WD). Larvae were reared on an artificial diet at $15^{\circ} \mathrm{C}$ and $12 \mathrm{~L}-12 \mathrm{D}$. Under these conditions, almost all pupae entered winter diapause. Puparia were maintained under the same conditions as larvae.

2) Summer diapause (SD). Larvae were reared at $25^{\circ} \mathrm{C}$ and $16 \mathrm{~L}-8 \mathrm{D}$. Under these conditions, more than $95 \%$ of pupae entered summer diapause. $\mathrm{Pu}-$ paria were maintained under the same conditions. At $25^{\circ} \mathrm{C}$, co-occurring non-diapausing pupae could be distinguished after day 8 by inspecting the pupal development through the semi-transparent pupal case, and were excluded from the sample. Summerdiapausing pupae before day 8 may have included at most 5\% non-diapausing individuals.

3) Non-diapause (ND). Larvae were reared at $23^{\circ} \mathrm{C}$ and $16 \mathrm{~L}-8 \mathrm{D}$. After pupariation, the insects were maintained at $17^{\circ} \mathrm{C}$ and $16 \mathrm{~L}-8 \mathrm{D}$ to advance non-diapause development in all pupae. At this temperature, no apparent adult morphogenesis occurred until day 14. Thus the 6-day-old pupae (ND6), which were subjected to cold- and hightemperature treatments, were completely indistinguishable from diapausing pupae.

To avoid a possible effect of temperature change, summer- and winter-diapausing pupae were maintained under the same conditions as larval rearing. In both types of diapause, diapause development (diapause ending process) progressed very slowly under the respective diapause-inducing conditions. None of the 10-day-old summer-diapausing pupae (SD10) and 40-day-old winter-diapausing pupae (WD40) completed diapause, however, a very small portion of SD40 and WD80 were considered to be in post-diapause development (Nomura and Ishikawa, 2000; Nomura, unpublished).

Cold-tolerance. A Petri dish containing 50 pupae (ND6, SD40, WD40 or WD80) was placed in a Styrofoam box and kept at $-15^{\circ} \mathrm{C}(-14.9 \pm$ $\left.0.2^{\circ} \mathrm{C}\right)$ or $-23^{\circ} \mathrm{C}\left(-22.9 \pm 0.3^{\circ} \mathrm{C}\right)$ for $2,4,6,9,12$ or 15 days, and then transferred to $23^{\circ} \mathrm{C}, 16 \mathrm{~L}-8 \mathrm{D}$. Adult eclosion was recorded for 20 days after the treatment. Then, all remaining pupae were inspected through the pupal case, and an individual was regarded dead if it was shrunken by desiccation. The individuals that had emerged as flies and 
those remaining as live pupae were regarded as survivors of the cold treatment.

High-temperature tolerance. More than 50 pupae each in summer diapause (SD10), winter diapause (WD10) or those not in diapause (ND6) were placed on a sheet of damp filter paper in a Petri dish and kept at $35 \pm 0.3^{\circ} \mathrm{C}$ for $2,4,6,9,12$ or 15 days, and then transferred to $23^{\circ} \mathrm{C}, 16 \mathrm{~L}-8 \mathrm{D}$. Twenty days after the treatment, the number of flies that had emerged was recorded and all remaining puparia were examined; a pupa was regarded as dead if it was shrunken. The individuals that emerged as flies and those remaining as live pupae were regarded as survivors of the high temperature treatment.

Reagents. Trehalase and $o$-phenylene diamine were obtained through Sigma (MO, U.S.A.) and Nacalai Tesque (Kyoto, Japan), respectively. Glucose oxidase and peroxidase were from Roche Diagnostics (Tokyo, Japan). Chloroform and glucose were purchased from Kanto Chemical Co. (Tokyo).

Trehalose content. The old cuticle of a puparium was removed carefully with forceps, and the pupa was homogenized in a micro-centrifuge tube with $600 \mu \mathrm{l}$ of $80 \%$ ethanol and centrifuged at $5,000 \mathrm{rpm}$ for $5 \mathrm{~min}$. The supernatant was transferred to a new micro-centrifuge tube, and dried in vacuo using a centrifugal evaporator (VEC-100, Asahi-Techno, Tokyo). Distilled water $(500 \mu \mathrm{l})$ and $200 \mu \mathrm{l}$ of chloroform were added to the residue and vortexed. After centrifugation at 3,000 rpm for 3 $\mathrm{min}$, the chloroform layer containing lipid was removed, and $500 \mu \mathrm{l}$ of distilled water was added. This preparation was stored at $-20^{\circ} \mathrm{C}$ until use for determination of trehalose content.

Trehalose in the sample was first converted to glucose by trehalase, and the amount of glucose produced was determined by the glucose oxidase method (Huggett and Nixon, 1957). Fifty microliters of the sample was incubated at $37^{\circ} \mathrm{C}$ for $1 \mathrm{~h}$ with or without $0.01 \mathrm{U}$ trehalase. After addition of $250 \mu \mathrm{l}$ glucose oxidase reagent $(0.25 \mathrm{M}$ phosphate buffer, 7.5 units glucose oxidase, 2.0 units peroxidase, $2.1 \mathrm{~mm} o$-phenylene diamine, $\mathrm{pH} 7.0$ ) and incubation at $30^{\circ} \mathrm{C}$ for $20 \mathrm{~min}$, sulfuric acid $(1.2 \mathrm{ml}$, $0.1 \mathrm{~N})$ was added to stop the reaction. Glucose concentration was calibrated by absorbance at $490 \mathrm{~nm}$ using standard glucose solutions. The amount of trehalose was obtained from $0.95 \times$ (amount of glucose in the sample treated with trehalase-that in the sample without trehalase).

\section{RESULTS}

\section{Cold hardiness}

A preliminary experiment showed that exposure to $-10^{\circ} \mathrm{C}$ for 15 days did not affect the survival of non-diapausing pupae, the survival rate being more than $95 \%$ (data not shown). At $-15^{\circ} \mathrm{C}$, the survival rate of 6-day-old non-diapausing pupae (ND6) after a 1 day exposure was $74 \%$, and decreased sharply as the duration of exposure was extended (Fig. 1). Only $2 \%$ of pupae could survive a 15 day exposure to $-15^{\circ} \mathrm{C}$. In contrast to the non-diapausing pupae, 40-day-old winter-diapausing pupae (WD40) were not affected by exposure to $-15^{\circ} \mathrm{C}$ for up to 15 days. Survival of 40-day-old summerdiapausing pupae (SD40) at $-15^{\circ} \mathrm{C}$ was also very high, although slightly lower than that of WD40. At $-23^{\circ} \mathrm{C}$, although survival of WD40 and 80-dayold winter-diapausing pupae (WD80) was not affected, the survival of SD40 decreased sharply when the duration of exposure exceeded 5 days (Fig. 2). These experiments indicated that the cold hardiness of onion fly pupae is in the following order; winter-diapausing pupae (WD40, WD80)> summer-diapausing pupae (SD40) $>$ non-diapausing pupae (ND6).

\section{High-temperature tolerance}

Survival of non-diapausing pupae after a $35^{\circ} \mathrm{C}$ treatment for up to 6 days was high (Fig. 3). How-

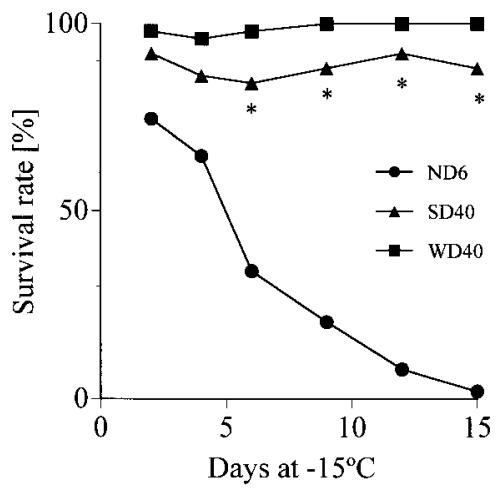

Fig. 1. Survival of Delia antiqua pupae after exposure to $-15^{\circ} \mathrm{C}$ for different periods $(n=50)$. After the exposure, pupae were maintained at $23^{\circ} \mathrm{C}$ and $16 \mathrm{~L}-8 \mathrm{D}$. ND6: 6-day-old non-diapausing pupae, SD40: 40-day-old summer-diapausing pupae, WD40: 40-day-old winter-diapausing pupae. * Significantly different from the survival of corresponding WD40 by Ryan's test on proportions (Ryan, 1960; $p<0.05$ ). 


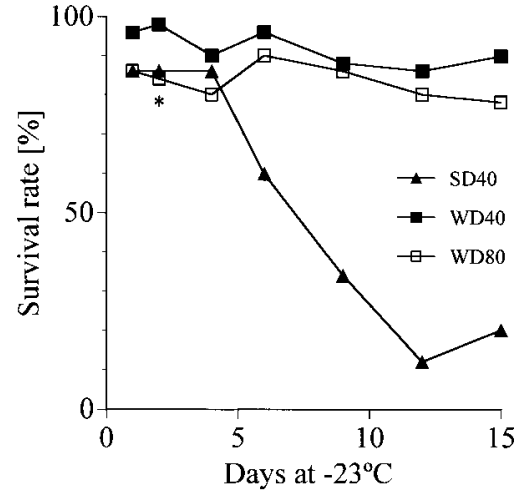

Fig. 2. Survival of Delia antiqua pupae after exposure to $-23^{\circ} \mathrm{C}$ for different periods. After the exposure, pupae were maintained at $23^{\circ} \mathrm{C}$ and $16 \mathrm{~L}-8 \mathrm{D}$. SD40: 40-day-old summerdiapausing pupae, WD40 and WD80: 40-day-old and 80-dayold winter-diapausing pupae, respectively. * Significantly different from the survival of corresponding WD40 by Ryan's test on proportions (Ryan, 1960; $p<0.05$ ).

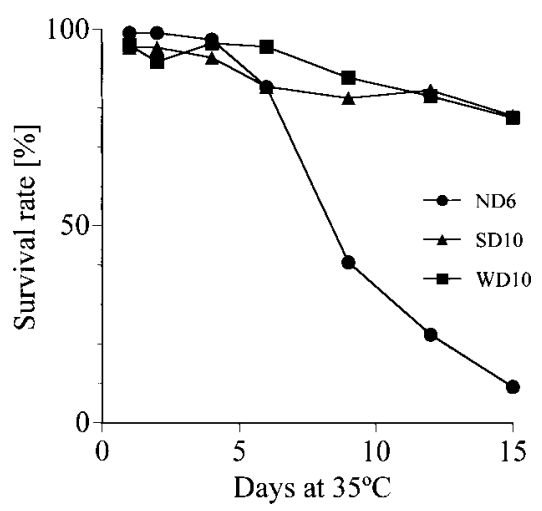

Fig. 3. Survival of Delia antiqua pupae after exposure to $35^{\circ} \mathrm{C}$ for different periods. After the exposure, pupae were maintained at $23^{\circ} \mathrm{C}$ and $16 \mathrm{~L}-8 \mathrm{D}$. ND6: 6-day-old non-diapausing pupae, SD10: 10-day-old summer-diapausing pupae, WD10: 10-day-old winter-diapausing pupae.

ever, only a few flies emerged during the 6-day treatment, although they were expected to emerge in 6 days at $35^{\circ} \mathrm{C}$ if pupal development had not been hindered. Since flies finally emerged from a large number of pupae when they were transferred to $23^{\circ} \mathrm{C}$, a transient disorder in pupal development caused by a short exposure to $35^{\circ} \mathrm{C}$ appears to be restorable. When the treatment at $35^{\circ} \mathrm{C}$ exceeded 6 days, however, the number of survivors decreased sharply (Fig. 3). Fewer flies emerged and there were no live pupae 20 days after the $35^{\circ} \mathrm{C}$ treatment. Less than $10 \%$ of non-diapausing pupae survived a 15 -day treatment at $35^{\circ} \mathrm{C}$. These results clearly indicate that $35^{\circ} \mathrm{C}$ is fatal for pupal devel-

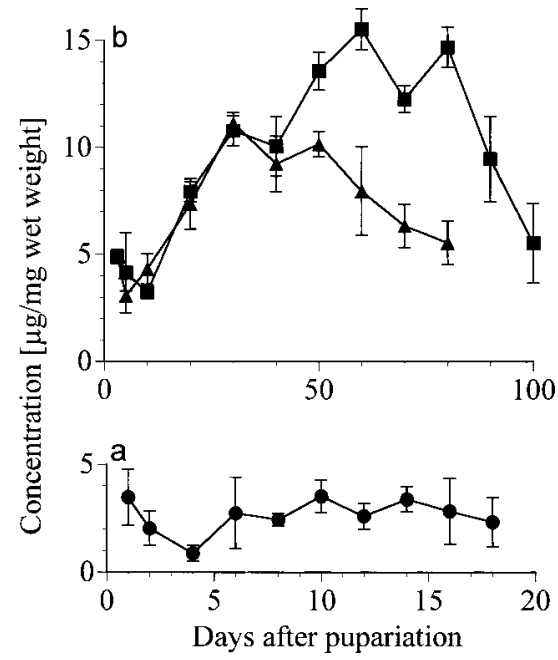

Fig. 4. Changes of trehalose content in non-diapausing, summer-diapausing and winter-diapausing Delia antiqua pupae $(n=4)$. a. $\bullet$ : non-diapausing pupae maintained at $17^{\circ} \mathrm{C}$ and $16 \mathrm{~L}-8 \mathrm{D}$. b. $\mathbf{\square}$ : winter-diapausing pupae maintained at $15^{\circ} \mathrm{C}$ and $12 \mathrm{~L}-12 \mathrm{D}, \mathbf{\Delta}$ : summer-diapausing pupae maintained at $25^{\circ} \mathrm{C}$ and $16 \mathrm{~L}-8 \mathrm{D}$. Bars indicate standard deviations.

opment in D. antiqua.

In contrast to non-diapause pupae, exposure to $35^{\circ} \mathrm{C}$ only marginally affected the survival of diapausing pupae (Fig. 3). The survival of summerand winter-diapausing pupae was greater than $75 \%$ even after a 15 -day treatment at $35^{\circ} \mathrm{C}$.

\section{Trehalose content}

Trehalose content of $D$. antiqua soon after pupariation was approximately $3-5 \mu \mathrm{g} / \mathrm{mg}$, regardless of diapause attributes (Fig. 4a, b). Trehalose content of non-diapausing pupae fluctuated in a small range during pupal development (Fig. 4a). After an initial small depression, the trehalose content in summer-diapausing pupae increased linearly and reached a maximum $(\approx 10 \mu \mathrm{g} / \mathrm{mg}) 30$ days after pupariation, and thereafter it decreased gradually (Fig. 4b). Although the trehalose content of winterdiapausing pupae followed a trend very similar to that of summer-diapausing pupae until day 40, it continued to increase until day 60 when it reached as high as $15 \mu \mathrm{g} / \mathrm{mg}$. The trehalose content of winter-diapausing pupae decreased sharply after day 80.

In summer- and winter-diapausing insects, it was obvious that trehalose accumulated after onset of diapause. It should also be noted that both summerand winter-diapausing pupae in the present study had never experienced a 'low' temperature; sum- 
mer- and winter-diapausing pupae were kept at $25^{\circ} \mathrm{C}$ and $16 \mathrm{~L}-8 \mathrm{D}$, and $15^{\circ} \mathrm{C}$ and $12 \mathrm{~L}-12 \mathrm{D}$, respectively. This finding indicates the presence of an underlying process to trehalose accumulation that does not require low temperature.

\section{DISCUSSION}

The most significant finding in the present study is the accumulation of trehalose in the summer-diapausing $D$. antiqua pupae, as well as in the winterdiapausing pupae. In a congener, $D$. radicum, winter-diapausing pupae accumulated trehalose, but pupae in high-temperature quiescence did not (Koštál and Šimek, 1995). These two congeners exhibit very similar dormancy at high temperatures. However, because of the difference in the requirement of diapause development, summer dormancy in $D$. antiqua is considered to be summer diapause (Ishikawa et al., 2000), and that in D. radicum is considered as high-temperature quiescence (Finch and Collier, 1985). Koštál and Šimek (1995) measured the trehalose content of quiescent pupae 7 days after pupariation at $27^{\circ} \mathrm{C}$. In $D$. antiqua, accumulation of trehalose became significant at day 20 (Fig. 4b). Thus the time of measurement in $D$. radicum may have been too early for a significant accumulation of trehalose. Alternatively, the difference in accumulation of trehalose in the summer dormancy in these two species may reflect the difference in the nature of dormancy.

It has been suggested that the origin of diapause is attributed to the tropical regions (Denlinger, 1986; Pullin and Wolda, 1993). Pullin (1996) supposed that metabolic suppression associated with diapause resulted in accumulation of low concentrations of carbohydrates, and that selection for enhanced carbohydrate accumulation occurred first in response to drought stress in tropical regions. The cryoprotective function of carbohydrates was selected for more recently to adapt to temperate and cold climates, allowing colonization of insects in these regions.

Accumulation of trehalose and enhanced tolerance of diapausing $D$. antiqua pupae may be explained in conjunction with the above hypothesis: Firstly, the metabolism in winter-diapausing pupae was actually shown to be extremely suppressed (Ishikawa et al., 1988). Secondly, the similar rate of trehalose accumulation in the two types of dia- pause suggests the same or a similar underlying mechanism. Lastly, cross-tolerance in D. antiqua diapausing pupae may be partly explained by the increase of trehalose, since this carbohydrate is known to be involved in various stress tolerance in several organisms (Romero et al., 1997; Soto et al., 1999; Guo et al., 2000).

The level of trehalose concentration in winter-diapausing $D$. antiqua pupae reached more than $1.5 \%$. Trehalose is considered to protect organisms from cold injury by stabilizing proteins and membrane lipids (Anchordoguy et al., 1987; Ring and Danks, 1998), and consequently increase overall cold hardiness. However, cold hardiness is not always strictly proportional to the concentration of trehalose. In D. radicum, the peak of trehalose content fluctuations did not coincide with the peak of cold hardiness (Koštál and Šimek, 1995). In D. antiqua, although trehalose concentrations in winterand summer-diapausing pupae at day 40 (WD40 and SD40) were similar $(\approx 10 \mu \mathrm{g} / \mathrm{mg})$, cold hardiness of these pupae differed significantly (WD40> SD40). These findings suggest that additional factor(s) are involved in the enhanced cold hardiness of $D$. antiqua and $D$. radicum diapausing pupae.

Survival of SD40 at $-15^{\circ} \mathrm{C}$ was slightly lower than that of WD40, however, this does not directly suggest that SD40 was less cold hardy than WD40. Since extension of cold treatment time apparently did not decrease the survival of pupae, the lower survival in SD40 can be attributed to the presence of less cold hardy heterogeneous pupae, which were probably in post-diapause development. The survival of WD80 at $-23^{\circ} \mathrm{C}$ was a little lower than that of WD40. This was also probably due to the presence of pupae that had completed diapause development. In this connection, the decrease in trehalose after day 40 in summer-diapausing pupae and after day 80 in winter-diapausing pupae may be related to completion of diapause development in an increasing number of pupae.

In $D$. antiqua, the upper threshold temperature for normal larval and pupal development appears to be less than $30^{\circ} \mathrm{C}$. At $30^{\circ} \mathrm{C}$, non-diapausing $D$. antiqua larvae and pupae could not complete development (Tomioka, 1977). Development of cyclorrhaphous fly puparia is divided into two distinct stages, the cryptocephalic and phanerocephalic stages (Fraenkel and Bhaskaran, 1973). Head evagination, which corresponds to 'pupation' 
in lepidopteran insects, divides these two stages. Under diapause-inducing conditions, development of puparia stops shortly after head evagination, i.e., at the beginning of phanerocephalic stage (Fraenkel and Hsiao, 1968; Ishikawa et al., 2000). In the present study, survival of non-diapausing pupae at $35^{\circ} \mathrm{C}$ decreased sharply when the treatment exceeded 6 days. It appears that non-diapausing pupae at the early phanerocephalic stage have superior high-temperature tolerance, however, it is lost rapidly as pupal development progresses. Since trehalose is not accumulated in non-diapausing pupae (Fig. 4a), the presence of early-phanerocephalic-stage specific factors that confer tolerance is strongly suggested.

D. antiqua is freeze-intolerant, that is, the insect cannot survive below its supercooling point. The supercooling point of pupae in the phanerocephalic stage is lower than $-28^{\circ} \mathrm{C}$ except for the last period when rapid adult morphogenesis is in progress (Ishikawa, unpublished data). This temperature is greater than $5^{\circ} \mathrm{C}$ below the lowest temperature examined in the present study. Thus, the cause of death after cold exposure treatments in the present study was not freezing, but cold injury. The changes in the supercooling points associated with the development of non-diapausing pupae, those associated with diapause and those associated with cold-acclimation have been investigated in detail and will be reported elsewhere.

The present study suggested that factor(s) other than accumulation of trehalose are involved in the enhanced tolerance of diapausing pupae. Further studies are required to clarify the mechanism for enhanced tolerance associated with summer and winter diapause.

\section{ACKNOWLEDGEMENTS}

We would like to express our thanks to Dr. S. Tatsuki and Mr. S. Hoshizaki of our laboratory for valuable advice and encouragement throughout the course of this study.

\section{REFERENCES}

Anchordoguy, T. J., A. S. Rudolph, J. F. Carpenter and J. H. Crowe (1987) Modes of interaction of cryoprotectants with membrane phospholipids during freezing. Cryobiology 24: 324-331.

D'amore, T., R. Crumplen and G. G. Stewart (1991) The involvement of trehalose in yeast stress tolerance. J. Indust. Microbiol. 7: 191-196.

Denlinger, D. L. (1986) Dormancy in tropical insects. Annu. Rev. Entomol. 31: 239-264.
Denlinger, D. L. (1991) Relationship between cold hardiness and diapause. In Insects at Low Temperature (D. L. Denlinger and R. E. Lee Jr. eds.). Chapman and Hall, New York, pp. 174-198.

Eleutherio, E. C. A., P. S. Araujo and A. D. Panek (1993) Protective role of trehalose during heat stress in Saccharomyces cerevisiae. Cryobiology 30: 591-596.

Finch, S. and R. H. Collier (1985) Laboratory studies on aestivation in the cabbage root fly (Delia radicum). Entomol. Exp. Appl. 38: 137-143.

Fraenkel, G. and G. Bhaskaran (1973) Pupariation and pupation in cyclorrhaphous flies (Diptera): terminology and interpretation. Ann. Entomol. Soc. Am. 66: 418-422.

Fraenkel, G. and C. Hsiao (1968) Manifestations of a pupal diapause in two species of flies, Sarcophaga argyrostoma and S. bullata. J. Insect Physiol. 14: 689-705.

Goto, M. and H. Hukushima (1995) Factors affecting the induction of summer and winter-diapause and diapause sensitive larval stage of cabbage-armyworm moth Manestra brassicae (Lepidoptera: Noctuidae). J. Yamagata Agric. For. Soc. 52: 17-24 (in Japanese).

Guo, N., I. Puhlev, D. R. Brown, J. Mansbridge and F. Levine (2000) Trehalose expression confers desiccation tolerance on human cells. Nature-Biotech. 18: 168-171.

Han, E.-N. and E. Bauce (1995) Glycerol synthesis by diapausing larvae in response to the timing of low temperature exposure, and implications for overwintering survival of the spruce budworm, Choristoneura fumiferana. J. Insect Physiol. 41: 981-985.

Huggett, A. S. G. and D. A. Nixon (1957) Enzymic determination of blood glucose. Biochem. J. 66: 12.

Ishikawa, Y., A. Mochizuki, T. Ikeshoji and Y. Matsumoto (1983) Mass-rearing of the onion and seed-corn flies, Hylemya antiqua and H. platura (Diptera: Anthomyiidae) on an artificial diet with antibiotics. Appl. Entomol. Zool. 18: 62-69.

Ishikawa, Y., K. Saito and Y. Matsumoto (1988) Carbon dioxide expiration rate of the onion fly, Hylemya antiqua Meigen (Diptera: Anthomyiidae) with reference to diapause. Appl. Entomol. Zool. 23: 194-196.

Ishikawa, Y., S. Tsukada and Y. Matsumoto (1987) Effect of temperature and photoperiod on the larval development and diapause induction in the onion fly, Hylemya antiqua Meigen (Diptera: Anthomyiidae). Appl. Entomol. Zool. 22: 610-617.

Ishikawa, Y., T. Yamashita and M. Nomura (2000) Characteristics of summer diapause in the onion maggot, Delia antiqua (Diptera: Anthomyiidae). J. Insect Physiol. 46: 161-166.

Kimura, Y. and S. Masaki (1992) Effect of light period on dark-time measurement for diapause induction in Mamestra brassicae. J. Insect Physiol. 38: 681-686.

Koštál, V. and P. Šimek (1995) Dynamics of cold hardiness, supercooling and cryoprotectants in diapausing and nondiapausing pupae of the cabbage root fly, Delia radicum. L. J. Insect Physiol. 41: 627-634.

Maki, Y., F. Yamaguchi and M. Yamashita (1959) Ecology and management of the onion maggot, Delia antiqua in Hyogo Prefecture, Japan. Agric. Hortic. 34: 957-960 (in 
Japanese).

Masaki, S. (1980) Summer diapause. Ann. Rev. Entomol. 25 : 1-25.

Nomura, M. and Y. Ishikawa (2000) Biphasic effect of low temperature on completion of winter diapause in the onion maggot, Delia antiqua. J. Insect Physiol. 46: 373377.

Park, C. G., J. S. Hyun, D. J. Cho and K. M. Choi (1990) Seasonal occurrence and summer diapause of the onion maggot, Delia antiqua (Meigen) (Diptera: Anthomyiidae). Korean J. Appl. Entomol. 29: 230-237 (in Korean with English summary).

Pullin, A. S. (1996) Physiological relationships between insect diapause and cold tolerance: Coevolution or coincidence? Eur. J. Entomol. 93: 121-129.

Pullin, A. S. and H. Wolda (1993) Glycerol and glucose accumulation during diapause in a tropical beetle. Physiol. Entomol. 18: 75-78.

Ring, R. A. and H. V. Danks (1998) The role of trehalose in cold hardiness and desiccation. Cryo-Letters 19: 275282.

Romero, C., J. M. Bellés, J. L. Vayá, R. Serrano, F. A. CuliáñezMacià (1997) Expression of the yeast trehalose- 6-phosphate synthase gene in transgenic tobacco plants: Pleiotropic phenotypes include drought tolerance. Planta 201: 293-297.

Ryan, T. A. (1960) Significance tests for multiple comparison of proportions, variances, and other statistics. Psychol. Bull. 57: 318-328.

Soto, T., J. Fernandez, S. J. Vicente, J. Cansado and M. Gacto (1999) Accumulation of trehalose by overexpression of tps1, coding for trehalose-6-phosphate synthase, causes increased resistance to multiple stresses in the fission yeast Schizosaccharomyces pombe. Appl. Environ. Microbiol. 65: 2020-2024.

Storey, J. M. and K. B. Storey (1983) Regulation of cryoprotectant metabolism in the overwintering gall fly larvae, Eurosta solidaginis: temperature control of glycerol and sorbitol levels. J. Comp. Physiol. 149: 495-502.

Tauber, M. J., C. A. Tauber and S. Masaki (1986) Seasonal Adaptations of Insects. Oxford University Press, New York. $411 \mathrm{pp}$.

Tomioka, T. (1977) Bionomics of the onion and seed-corn flies, Hylemya antiqua and H. platura. Plant Protec. 31: 206-209 (in Japanese). 